\title{
Doping and temperature dependence of thermoelectric properties in $\mathrm{Mg}_{2}(\mathrm{Si}, \mathrm{Sn})$
}

\author{
J. J. Pulikkotil, ${ }^{1}$ D. J. Singh, ${ }^{2}$ S. Auluck, ${ }^{1}$ M. Saravanan, ${ }^{1}$ D. K. Misra, ${ }^{1}$ A. Dhar,${ }^{1}$ and R. C. Budhani ${ }^{1}$ \\ ${ }^{1}$ Council of Scientific and Industrial Research—National Physical Laboratory, New Delhi 110012, India \\ ${ }^{2}$ Materials Science and Technology Division, Oak Ridge National Laboratory, Oak Ridge, Tennessee 37831-6056, USA
}

(Received 26 May 2012; revised manuscript received 25 July 2012; published 4 October 2012)

\begin{abstract}
We report the use of Boltzmann transport theory to investigate the electrical properties of thermoelectric $\mathrm{Mg}_{2} \mathrm{Si}, \mathrm{Mg}_{2} \mathrm{Sn}$, and a supercell model of the 50-50 alloy. The results are based on first-principles electronic structure calculations with the modified Becke-Johnson potential of Tran and Blaha, which yields band gaps in good accord with experiment. The calculated transport coefficients are discussed in relation to the thermoelectric performance of these materials. The results imply roughly symmetric behavior with respect to carrier type and the possibility of improvements in $Z T$, especially for $p$-type and lower temperatures.
\end{abstract}

DOI: 10.1103/PhysRevB.86.155204

PACS number(s): 72.20.Pa, 71.20.Lp

\section{INTRODUCTION}

Thermoelectric materials allow the direct solid-state conversion between electrical and thermal energy. ${ }^{1,2}$ They are of current interest for a number of energy related applications, such as waste heat recovery and solar thermal devices. ${ }^{3,4}$ Thermoelectric performance is characterized by a dimensionless figure of merit, $Z T=\sigma S^{2} T / \kappa$, where $\sigma$ is the electrical conductivity, $S$ is the thermopower, $T$ is temperature, and $\kappa=\kappa_{l}+\kappa_{e}$ is the thermal conductivity, which as normal is written as a sum of lattice and electronic contributions. While there is no known fundamental limit on $Z T$, for many years the highest known values in existing materials were $\sim 1-\mathrm{a}$ limit that is now approaching $\sim 2$ due to recent discoveries, e.g., in skutterudites and chalcogenides. ${ }^{5-7}$ This fact, and the counterindication of the transport properties comprising $Z T$ (e.g., high $\sigma$ but low $\kappa$ and high $S$ and high $\sigma$ ), have led to substantial interest in thermoelectrics from a fundamental point of view.

Returning to applications, $Z T$ limits the efficiency of thermoelectric converters, and therefore high $Z T$ is important. In practice, it is also helpful to have nontoxic materials composed of earth abundant, and therefore available, elements. One such material is $\mathrm{Mg}_{2} \mathrm{Si}$. $Z T$ values exceeding unity have been found in various $\mathrm{Mg}_{2} X(X \equiv \mathrm{Si}, \mathrm{Ge}, \mathrm{Sn})$ based alloys. ${ }^{8-10}$ These materials have attracted much recent attention. ${ }^{10-24} \mathrm{~A}$ particularly interesting potential application is in exhaust gas waste heat recovery in vehicles, where the environmental friendliness of silicide alloys compared to alternative high performance materials such as $\mathrm{Pb}$ chalcogenides is very important, and where a high $Z T$ over the temperature range 400-600 K would be desirable. ${ }^{25}$

One issue with $\mathrm{Mg}_{2} X$ thermoelectrics is that they are difficult to optimize experimentally because the high vapor pressure of $\mathrm{Mg}$ complicates controlled synthesis. Nonetheless, optimization of the doping level is essential for high $Z T$. This is well illustrated by recent results for $\mathrm{PbSe}$, which was long thought to be a rather poor thermoelectric, but now has been shown to be a very good material with proper doping. ${ }^{7,26}$ Optimization of doping is critical for characterizing the performance of a thermoelectric material. Here we use first-principles calculations and Boltzmann transport theory to address this for $\mathrm{Mg}_{2} \mathrm{Si}$, and the alloy with $\mathrm{Sn}$, specifically the extent to which it is already optimized with respect to carrier concentration for both $p$ and $n$ types, and second to what extent performance in the $400-600 \mathrm{~K}$ range can be improved by optimization for that range, as opposed to the normal focus on increasing the peak value of $Z T$ at any temperature (i.e., in the present case $\sim 700-800 \mathrm{~K}$ ).

Various doping strategies have been used for $\mathrm{Mg}_{2} \mathrm{Si}$. $p$-type materials have been made using $\mathrm{Cu}$ and $\mathrm{Ag}$ at the $\mathrm{Mg}$ site $^{27,28}$ and also by $\mathrm{Ga}$ on the $\mathrm{Si}$ sites. ${ }^{23} n$-type dopants include $\mathrm{Al}, \mathrm{Sc}$, and $\mathrm{Y}$ at the $\mathrm{Mg}$ site $^{29}$ and $\mathrm{P}, \mathrm{Se}, \mathrm{Sb}, \mathrm{Te}$, and $\mathrm{Bi}$ at the $\mathrm{Si}$ sites. ${ }^{11,20,21} \mathrm{ZT}$ values of 0.8 at $\sim 800 \mathrm{~K}$ were reported in $\mathrm{Bi}$ doped $\mathrm{Mg}_{2} \mathrm{Si}^{14}$ Partial substitution of $\mathrm{Bi}$ with $\mathrm{Al}$ in $\mathrm{Mg}_{2}\left(\mathrm{Si}_{1-x} \mathrm{Bi}_{x}\right)$ was found to further improve the thermoelectric properties. ${ }^{11}$ Further, isoelectronic substitution at the $\mathrm{Si}$ site in $\mathrm{Mg}_{2} \mathrm{Si}$ with $\mathrm{Ge}$ and $\mathrm{Sn}$ also enhanced the $Z T$ value. For example, in the $n$-type $\operatorname{Mg}_{2}\left(\operatorname{Si}_{1-x} \operatorname{Sn}_{x}\right)$ alloys, with carrier concentration $\sim 10^{20} \mathrm{~cm}^{-3}$, the $Z T$ was estimated to be $\sim 1.1$, in the composition range $0.4 \leqslant x \leqslant 0.6{ }^{8}$ With further increase in the carrier concentration accomplished by doping 2.5 at. $\%$ of $\mathrm{Bi}$ in the disordered $\mathrm{Mg}_{2}\left(\mathrm{Si}_{1-x} \mathrm{Sn}_{x}\right)$ alloys, the $Z T$ decreases to 0.78 at $800 \mathrm{~K} .{ }^{13}$ Similarly, notable changes in the $Z T$ parameter were also observed in the $S n$ and Sb doped alloys. ${ }^{10,15,16,19}$ The maximum $Z T$ values $(\geqslant 1.2)$ reported are for $n$-type alloys in the range $x \sim 0.5-0.7 .^{8-10,12}$ In practice, $p$ - and $n$-type materials with good performance are both needed for application. We note that the performance of $n$-type $\mathrm{Mg}_{2}(\mathrm{Si}, \mathrm{Sn})$ has been discussed in terms of a band crossing as a function of the $\mathrm{Si}-\mathrm{Sn}$ composition, most recently by Liu and co-workers. ${ }^{12}$

Here, we address the doping dependence of thermoelectric properties in the $\mathrm{Mg}_{2} \mathrm{X}$ compounds and in its solid solution, viz. $\mathrm{Mg}_{2}\left(\mathrm{Si}_{0.5} \mathrm{Sn}_{0.5}\right)$. The calculations are based on the application of Boltzmann transport theory to the electronic structure, which we determined using a recently developed modification of the Becke-Johnson ${ }^{30}$ potential due to Tran and Blaha (denoted TB-mBJ). ${ }^{31}$ This functional provides band gaps in good accord with experiment for simple semiconductors and insulators. ${ }^{32-38}$ This is important for predicting thermoelectric behavior.

\section{COMPUTATIONAL METHODS}

The present calculations were performed using the general potential linearized augmented plane-wave (LAPW) method ${ }^{39}$ as implemented in the WIEN2K code. ${ }^{40}$ The equilibrium lattice 
constants were determined using the generalized gradient approximation (GGA) of Perdew, Burke, and Ernzerhof. ${ }^{41} \mathrm{We}$ used a Brillouin-zone sampling based on $216 k$ points in the irreducible wedge of the Brillouin zone for the total-energy calculations, and much denser grids ( $\sim 6000 k$ points $)$ for the transport calculations. We used well-converged basis sets with $R_{\mathrm{MT}} K_{\max }=8$ where $R_{\mathrm{MT}}$ and $K_{\max }$ are the smallest muffin-tin radius and the maximum size of reciprocal-lattice vectors, respectively. The LAPW sphere radii for $\mathrm{Mg}$ and $X$ ions were $2.2 a_{0}$. We treated the core electrons relativistically, and included relativity at the scalar relativistic level for the valence electrons. Importantly, as mentioned, the electronic structures were obtained using the recently developed TB-mBJ functional, ${ }^{31}$ which greatly improves the band structures, and particularly the band gaps of materials like $\mathrm{Mg}_{2} \mathrm{Si}$. This allows calculation of band-structure related quantities without adjustment.

The transport properties were calculated from the electronic structure using Boltzmann theory and the constant scattering time approximation (CSTA) as implemented in the BOLTZTRAP code. $^{42}$ The CSTA, which has been successfully applied to a wide range of thermoelectric materials, ${ }^{43}$ allows for direct calculation of the Seebeck coefficient as a function of temperature and doping level, with no adjustable parameters. $\mathrm{Mg}_{2}\left(\mathrm{Sn}_{0.5} \mathrm{Si}_{0.5}\right)$ was modeled using a supercell. The lattice parameters and internal coordinates in the supercell were determined by total-energy minimization.

\section{RESULTS AND DISCUSSION}

For $\mathrm{Mg}_{2} \mathrm{Si}$ and $\mathrm{Mg}_{2} \mathrm{Sn}$, the equilibrium lattice constants $\left(\mathrm{a}_{0}\right)$ computed by means of GGA total-energy minimization were determined as 6.364 and $6.805 \AA$, respectively, while their corresponding bulk modulus was determined to be 54 and $42 \mathrm{G} \mathrm{Pa}$, respectively, in comparison with experiment $\left(\mathrm{a}_{0} \simeq 6.39 \AA\right.$ and $\mathrm{B}_{0} \simeq 55 \mathrm{G} \mathrm{Pa}$ for $\mathrm{Mg}_{2} \mathrm{Si}$ and $\mathrm{a}_{0} \simeq 6.66 \AA$ and $\mathrm{B}_{0} \simeq 41 \mathrm{G} \mathrm{Pa}$ for $\left.\mathrm{Mg}_{2} \mathrm{Sn}\right){ }^{44}$ Thus, besides the higher density, $\mathrm{Mg}_{2} \mathrm{Sn}$ is also softer than $\mathrm{Mg}_{2} \mathrm{Si}$, which from the point of view of thermal conductivity means that, in addition to scattering due to mass disorder in alloys, one can also expect force constant disorder. We obtain $\mathrm{a}_{0}=6.594 \AA$ and $\mathrm{B}_{0}=$ $47 \mathrm{G} \mathrm{Pa}$ for our $\mathrm{Mg}_{2}\left(\mathrm{Si}_{0.5} \mathrm{Sn}_{0.5}\right)$ supercell.

The calculated density of states (DOS) and band structures of $\mathrm{Mg}_{2} \mathrm{Si}$ and $\mathrm{Mg}_{2} \mathrm{Sn}$ at their theoretically optimized equilibrium lattice constants are shown in Figs. 1 and 2, respectively. The results with the PBE-GGA show that the GGA not only underestimates the band gap for $\mathrm{Mg}_{2} \mathrm{Si}$, but also incorrectly predicts $\mathrm{Mg}_{2} \mathrm{Sn}$ to be a metal. The TB-mBJ potential gives very much improved band-gap values of 0.6 and $0.3 \mathrm{eV}$, for $\mathrm{Mg}_{2} \mathrm{Si}$ and $\mathrm{Mg}_{2} \mathrm{Sn}$, respectively. These are consistent with the experimental values and a recent calculation for $\mathrm{Mg}_{2} \mathrm{Si}$ using this functional by Boulet and Record. ${ }^{45}$

The electrical transport quantities, $\sigma, S$, and $\kappa_{e}$, are in general functions of doping and temperature that depend in detail on the band structure. Electrical and optical experiments characterize $\mathrm{Mg}_{2} X(X=\mathrm{Si}, \mathrm{Ge}$, and $\mathrm{Sn})$ compounds as indirect band-gap semiconductors ${ }^{46-48}$ with gap magnitudes of $\sim 0.7 \mathrm{eV}$ for $\mathrm{Mg}_{2} \mathrm{Si}^{46} \sim 0.6 \mathrm{eV}$ for $\mathrm{Mg}_{2} \mathrm{Ge},{ }^{46,49}$ and $\sim 0.3 \mathrm{eV}$ for $\mathrm{Mg}_{2} \mathrm{Sn} .{ }^{50}$ First-principles calculations also predict indirect band gaps. ${ }^{51,52}$ As mentioned, band gaps are underestimated

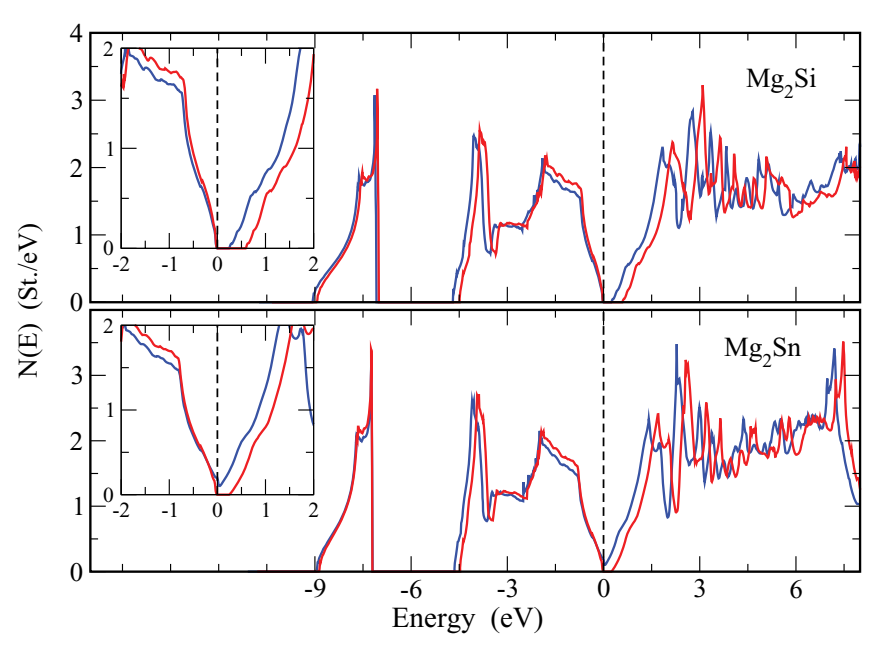

FIG. 1. (Color online) Comparison of the PBE-GGA (blue curves) and TB-mBJ (red curves) electronic density of states of $\mathrm{Mg}_{2} \mathrm{Si}$ (upper panel) and $\mathrm{Mg}_{2} \mathrm{Sn}$ (lower panel). The densities of states are plotted on a per formula unit basis. Insets: blowups near the band edge. The energy zero (vertical dashed line) is at the valence-band maximum.

with standard functionals. However, the valence bandwidths are in good accord with experiment. ${ }^{51,52}$ Arnaud and Alouani ${ }^{53}$ reported calculations based on the $G W$ approximation, showing that these band-gap errors are largely corrected in $\mathrm{Mg}_{2} \mathrm{Si}$ and $\mathrm{Mg}_{2} \mathrm{Ge}$.

The general features of our band structures are similar to those in previous reports, ${ }^{53-56}$ with the exception that the band gap is improved as expected from the use of the TB-mBJ functional. As seen in Figs. 1 and 2, we find that the overall features of the valence bands of the two compounds are similar. They are mainly derived from the $\mathrm{Si}(\mathrm{Sn}) p$ states. Both compounds have an indirect gap from $\Gamma$ to $X$ and $\Gamma$ to $K$ of the fcc Brillouin zone.

The states making up the bottom of the conduction band are, however, different in these isoelectronic compounds. In $\mathrm{Mg}_{2} \mathrm{Si}$, the lowest conduction band is a hybridized $\mathrm{Mg}-3 s-\mathrm{Si}-3 p$ band (at the $X$ and $K$ points) followed by $\mathrm{Mg}$, a mainly $3 s$ band $\sim 0.18 \mathrm{eV}$, above it. In $\mathrm{Mg}_{2} \mathrm{Sn}$, this structure of the two lowest conduction bands at the the $X$ and $K$ points is reversed. This is because the unoccupied $\mathrm{Mg}$ bands are lower in energy in comparison to the $\mathrm{Sn}$ bands in $\mathrm{Mg}_{2} \mathrm{Sn}$, while the unoccupied $\mathrm{Mg} 3 s$ and $\mathrm{Si} 3 p$ states are almost degenerate in $\mathrm{Mg}_{2} \mathrm{Si}$. The hybridized $\mathrm{Mg}-3 s-\mathrm{Sn}-5 p$ bands in $\mathrm{Mg}_{2} \mathrm{Sn}$ are $0.31 \mathrm{eV}$ above the lowest $\mathrm{Mg}-3 s$ band. This is the composition dependent band crossing mentioned by Zaitsev and co-workers ${ }^{8}$ and subsequently emphasized by Liu and co-workers, ${ }^{12}$ who both reported similar high values of $Z T$ in $n$-type $\operatorname{Mg}_{2}(\mathrm{Si}, \mathrm{Sn})$ alloys. Zaitsev and co-workers also emphasize however the importance of reduction in thermal conductivity upon alloying. ${ }^{8}$ We emphasize that there is a composition dependent band crossing for the conduction bands, but that the valence-band structure is different, showing a mixture of heavy and light bands at the valence-band maximum, but no composition dependent crossover.

In general, $s$ and $p$ orbitals are rather extended and overlap between sites. This can make the assignment of 


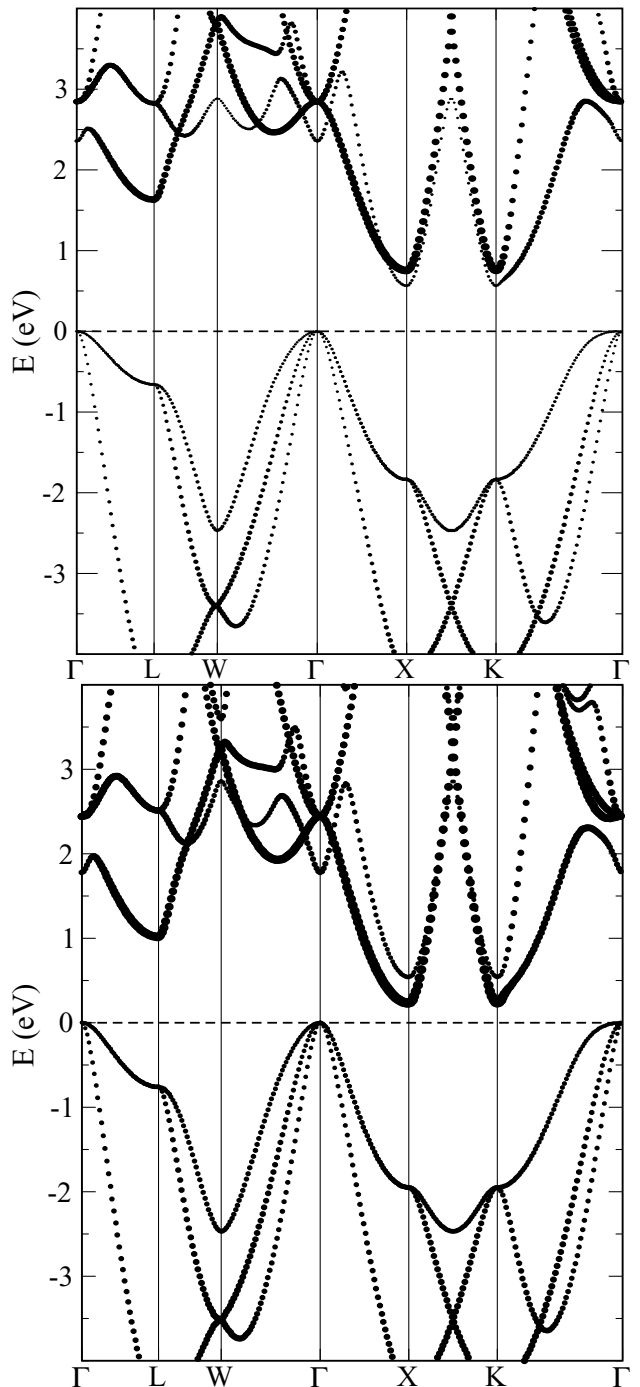

FIG. 2. Band structure of $\mathrm{Mg}_{2} \mathrm{Si}$ and $\mathrm{Mg}_{2} \mathrm{Sn}$ at their equilibrium lattice constants, with the fat bands representing $\mathrm{Mg}$ (i.e., the plotting symbol size gives the contribution from $\mathrm{Mg}$ derived states). The dashed horizontal line at $0 \mathrm{eV}$ shows the valence-band maximum.

orbital character to band states ambiguous. However, this can be addressed in part by projecting onto small spheres, as was discussed in the context of hydrides. ${ }^{57,58}$ This can give information about the relative contribution of a certain orbital character to different bands or energy regions. For this purpose, we did additional calculations using smaller $\mathrm{Mg}$ and Si LAPW spheres of radius $1.8 a_{0}$. The relative $\mathrm{Mg}$ contributions to the density of states for $\mathrm{Mg}_{2} \mathrm{Si}, \mathrm{Mg}_{2} \mathrm{Sn}$, and the 50-50 supercell are given in Fig. 3. As may be seen, the two compounds and the supercell are very similar in the energy dependent distribution of $\mathrm{Mg}$ contribution to the DOS. Figure 4 shows projections onto small $\mathrm{Mg}$ and $\mathrm{Si}$ spheres for $\mathrm{Mg}_{2} \mathrm{Si}$ over an expanded energy range. As may also be seen, the $\mathrm{Si} 3 s$ states form a narrow band at high binding energy, $\sim 8 \mathrm{eV}$ below the valence-band maximum. The valence and conduction bands are derived primarily from hybridized $\mathrm{Mg}$ $2 s$ and $\mathrm{Si} 3 p$ orbitals. The valence bands have stronger $\mathrm{Si}$ $3 p$ character, while the conduction bands have stronger $\mathrm{Mg}$ character, indicating charge transfer from $\mathrm{Mg}$ to $\mathrm{Si}$, consistent

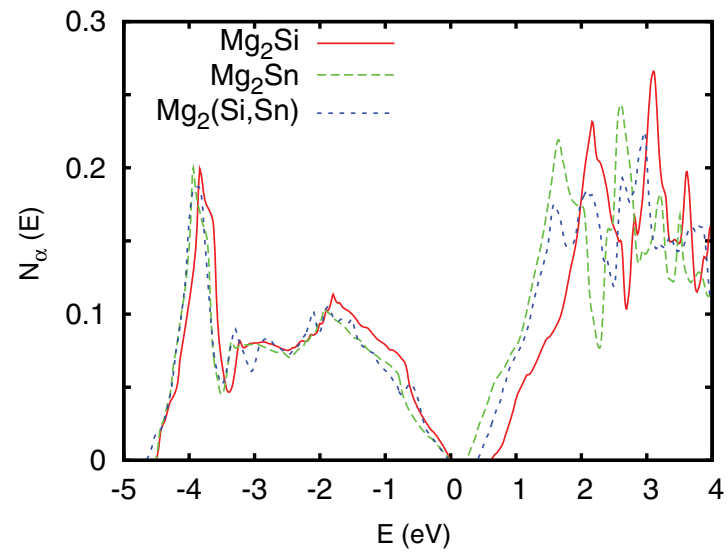

FIG. 3. (Color online) Electronic densities of states projected onto a small $\mathrm{Mg}$ sphere, radius $1.8 a_{0}$. The result is shown per formula unit.

with the fact that $\mathrm{Si}$ is substantially more electronegative than $\mathrm{Mg}$.

We note that the formation of the electronic structure is quite different from that in elemental $\mathrm{Si}$. In $\mathrm{Si}$, the band structure reflects the $s p^{3}$ hybrid orbital covalent bonding, with the eight valence electrons per unit cell (two $\mathrm{Si}$ atoms) occupying the nominally $s p^{3}$ bonding valence bands, and with the conduction bands formed by the corresponding antibonding combinations. In $\mathrm{Mg}_{2} \mathrm{Si}$ such hybrid orbitals are not formed, and instead the $\mathrm{Si}$ $3 s$ orbitals are at high binding energy, forming, as mentioned, a narrow band $\sim 8 \mathrm{eV}$ below the valence-band maximum. The valence band is formed from $\mathrm{Si} p$ states (hybridized with $\mathrm{Mg} s$ ) and is rather narrow, with a total width of slightly more than $4 \mathrm{eV}$. The electron counting is such that each $\mathrm{Mg}$ contributes two valence electrons and each Si contributes two $p$ electrons and two $s$ electrons. As mentioned, the $\mathrm{Si} s$ band stays at high binding energy below the $p$-like valence bands. With two $\mathrm{Mg}$ per formula unit, one then has the two $s$ electrons, plus six additional valence electrons, which can completely fill the $p$-like valence band. The density of states reflects this electron counting. However, it is important to emphasize that there is hybridization between $\mathrm{Mg} s$ and $\mathrm{Si} p$ states. This can be seen in the $\mathrm{Si}$ character present in the conduction bands, as well

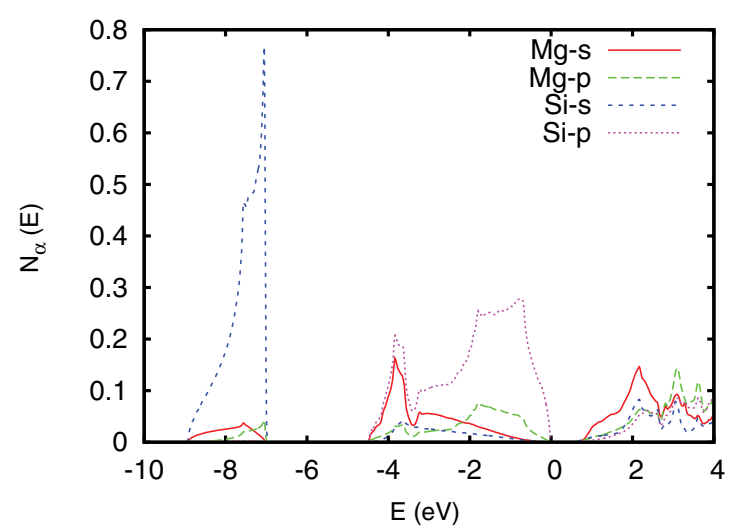

FIG. 4. (Color online) Projections of the electronic density of states for $\mathrm{Mg}_{2} \mathrm{Si}$ onto different orbitals with small $\mathrm{Mg}$ and $\mathrm{Si}$ sphere radii of $1.8 a_{0}$. The result is shown per formula unit. 
as in the rather prominent $\mathrm{Mg}$ character in the very bottom of the $\mathrm{Si} p$ derived valence band, which reflects $\sigma$ bonding Si- $p-\mathrm{Mg}-s$ combinations (see Fig. 4).

The valence-band maximum is made up by a triply degenerate band at the $\Gamma$ point. Away from $\Gamma$ these split into a light band, and two heavier bands, which are approximately parabolic near $\Gamma$ but become more weakly dispersive as one moves away from the zone center, depending on the specific direction (see, e.g., $\Gamma-L$ ). The lower light band takes a nearly linear dispersion starting rather close to $\Gamma$ : e.g., within $\sim 3-4 \%$ of the distance to the Brillouin-zone boundary ( $L$ point), corresponding to hole doping levels of $\sim 2 \times 10^{-3}$ holes/f.u., in both materials. The slopes of this band are -14 and $-15 \mathrm{eV} \AA$ for $\mathrm{Mg}_{2} \mathrm{Si}$ and $\mathrm{Mg}_{2} \mathrm{Sn}$, respectively. Thus the valence bands of importance for $p$-type doping show a combination of heavy and light bands, which has been previously discussed as favorable for thermoelectric performance. ${ }^{26,59}$ One can also see this in the DOS, which increases rapidly away from the band edge for both the valence and conduction bands, meaning that as far as the increase of the DOS away from the band edge, both the conduction and valence bands show heavy features that all things being equal would be similarly favorable for thermoelectric performance.

As mentioned, to date the best $n$-type thermoelectric performance has been found in disordered $\mathrm{Mg}_{2}\left(\mathrm{Si}_{1-x} \mathrm{Sn}_{x}\right)$ alloys in the concentration range $0.4<x<0.7$. This may be partly associated with the chemical disorder, which usually tends to decrease thermal conductivity, ${ }^{9}$ and also band structure tuning, as mentioned. We did supercell calculations for $x=0.5$. The results shown are for a simple ordering based on the original cubic structure. Specifically, the cell was doubled to a $P 4 / \mathrm{mmm}$ structure with $\mathrm{Si}$ at $(0,0,0)$, Sn at $\left(\frac{1}{2}, \frac{1}{2}, \frac{1}{2}\right)$, and $\mathrm{Mg}$ on the sites $\left(0, \frac{1}{2}, z\right)$, with the relaxed $z=0.2394$ (formula, $\mathrm{Mg}_{4} \mathrm{SiSn}$ ). We also tested other choices. While, as is generally true, zone folding produces superficially complex band structures in supercells, we find that the physical properties are little changed in this system. For example, we also did calculations for a more complex lower symmetry cell (24 atoms, space group $P m$ ), again with structural relaxation. The structures and electronic DOS for the two cells are shown in Fig. 5. As may be seen, on a per formula unit basis, the shapes of the DOS overall, and importantly near the band edges for the two cells, are very similar. Therefore, for this particular material, we can use a simple supercell to study electronic structure dependent properties.

The band gap in our supercell was $0.41 \mathrm{eV}$, which is between the calculated values for the end-point compounds. Also the separation of the two bands making up the conduction-band edge is reduced now to $0.04 \mathrm{eV}$.

We now turn to the transport properties. Calculated transport coefficients $S$ and $\sigma S^{2} / \tau$ of $\mathrm{Mg}_{2} \mathrm{Si}$ and $\mathrm{Mg}_{2} \mathrm{Sn}$ as functions of doping level at various temperatures are given in Figs. 6 and 7, respectively. The calculation results for the 50-50 supercell are shown in Fig. 8. The variations in the theoretical Seebeck coefficients $S(T)$ for both $n$-type and $p$-type doping in $\mathrm{Mg}_{2} \mathrm{Si}$ for low carrier concentrations are in accord with experimental results ${ }^{23}$ as are values for higher carrier concentrations characteristic of thermoelectric samples.

There is a clear bipolar suppression of the thermopower in $\mathrm{Mg}_{2} \mathrm{Sn}$ for both $p$ type and $n$ type at low carrier concentrations.
A

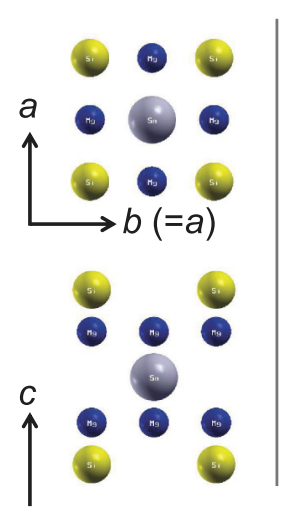

B
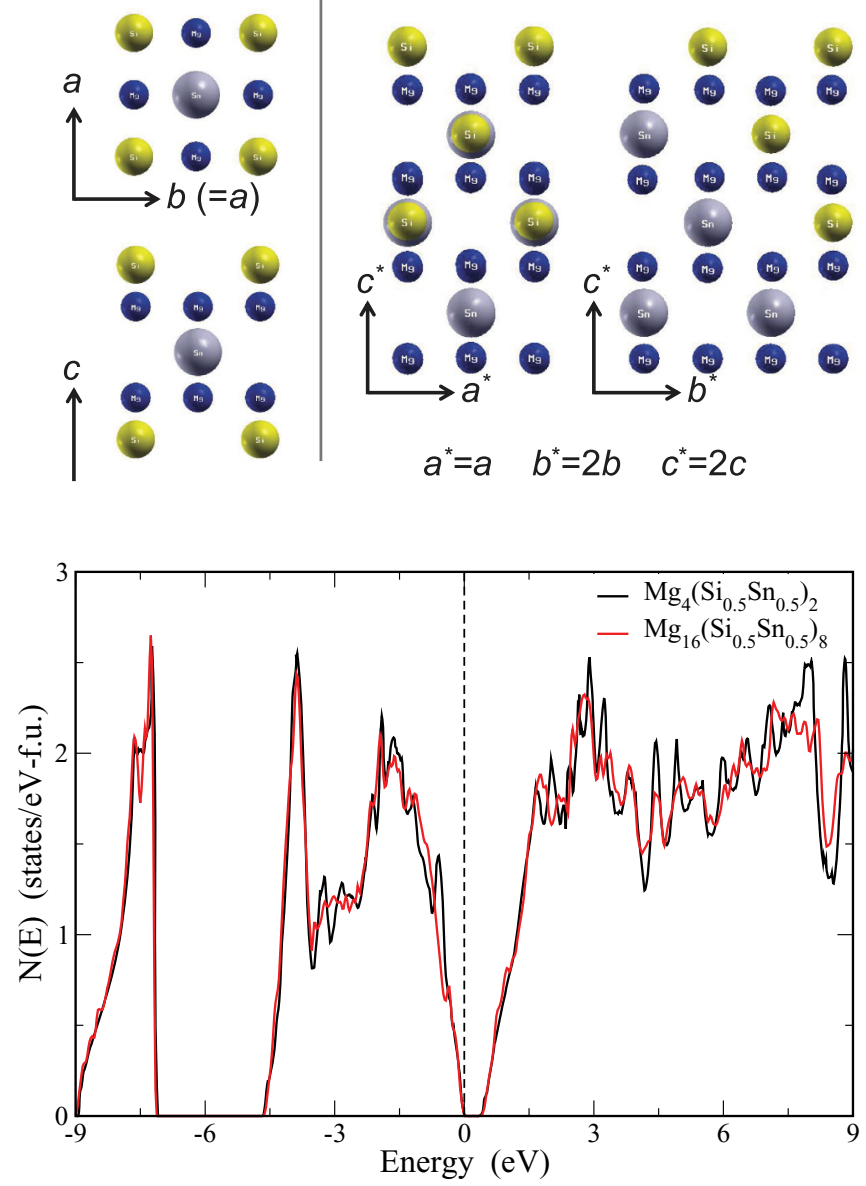

FIG. 5. (Color online) Structure of two supercells (top); compositions $\mathrm{A}=\mathrm{Mg}_{4}\left(\mathrm{Si}_{0.5} \mathrm{Sn}_{0.5}\right)_{2}$ and $\mathrm{B}=\mathrm{Mg}_{16}\left(\mathrm{Si}_{0.5} \mathrm{Sn}_{0.5}\right)_{8}$, showing $\mathrm{Mg}$ as small blue spheres, $\mathrm{Si}$ as medium gold spheres, and $\mathrm{Sn}$ as large gray spheres; and comparison of the electronic DOS for the two 50-50 supercells (bottom, see text). Densities of states are shown per $\mathrm{Mg}_{2}\left(\mathrm{Si}_{0.5} \mathrm{Sn}_{0.5}\right)$ formula unit. The energy zero (vertical dashed line) is at the valence-band maximum.

In fact, this behavior is rather symmetric in carrier type, reflecting the similar shapes of the density of states for the valence and conduction bands. However, in detail, the maximum $p$-type thermopowers for a given $T$ are higher than the maximum $n$-type thermopowers in $\mathrm{Mg}_{2} \mathrm{Sn}$.

This bipolar effect is not evident for $\mathrm{Mg}_{2} \mathrm{Si}$ over almost all the $T$ and carrier-concentration range shown in Fig. 6, the exception being the downward curvatures in the $750-\mathrm{K}$ curve for carrier concentrations below $\sim 2 \times 10^{19} \mathrm{~cm}^{-3}$, which is stronger for $p$ type. The weaker bipolar effect in $\mathrm{Mg}_{2}$ Si relative to $\mathrm{Mg}_{2} \mathrm{Sn}$ is a simple consequence of the larger band gap of the silicide. The behavior of the 50-50 supercell (Fig. 8) is intermediate between that of $\mathrm{Mg}_{2} \mathrm{Si}$ and $\mathrm{Mg}_{2} \mathrm{Sn}$. In particular, the supercell still shows a bipolar effect, though reduced compared to $\mathrm{Mg}_{2} \mathrm{Sn}$. Also, the values of the thermopower for a given carrier concentration in the supercell are generally higher than for $\mathrm{Mg}_{2} \mathrm{Sn}$, and the magnitudes for $p$ type and $n$ type are more similar, as in $\mathrm{Mg}_{2} \mathrm{Si}$.

A key point is that in $\mathrm{Mg}_{2} \mathrm{Si}$ the behavior and magnitudes of the thermopowers as functions of carrier concentration over 

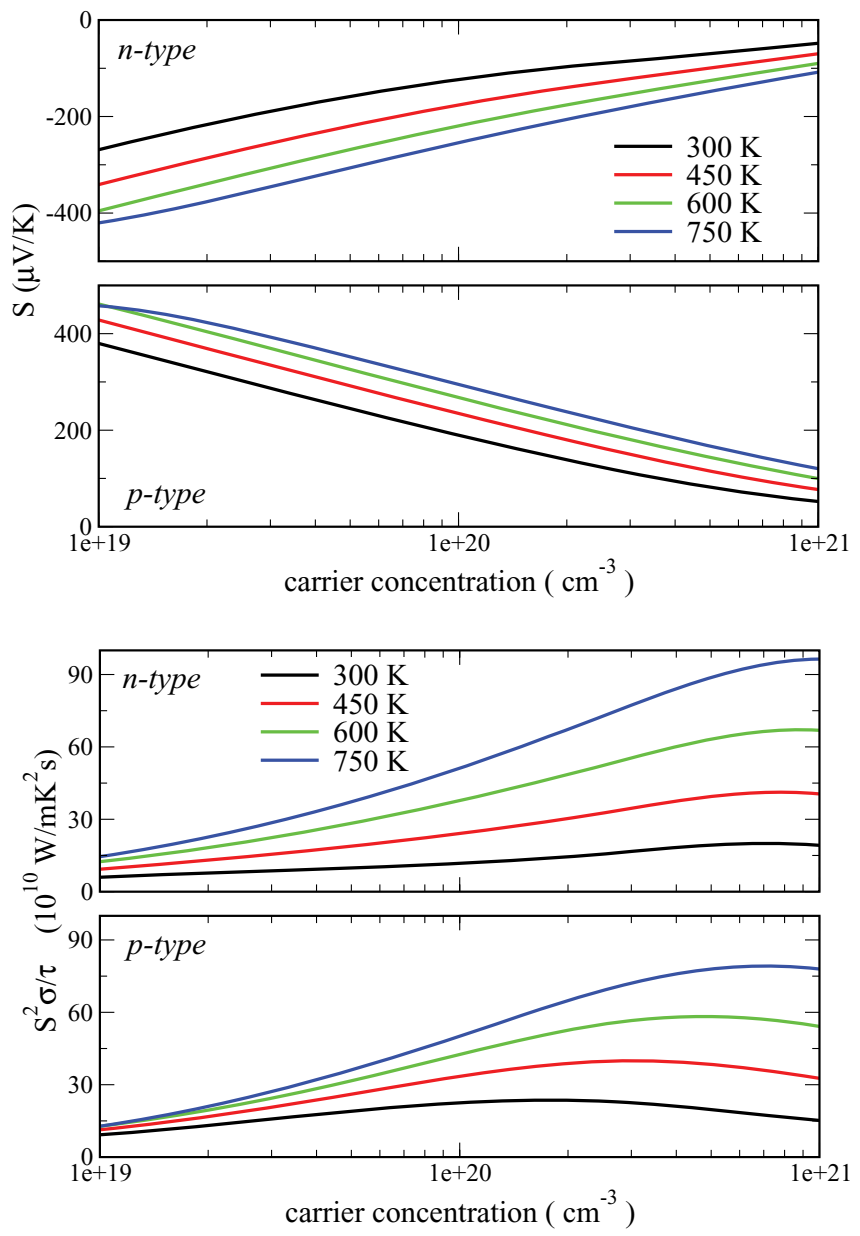

FIG. 6. (Color online) Calculated thermoelectric properties of $\mathrm{Mg}_{2} \mathrm{Si}$ for both $p$-type and $n$-type charge carriers, where $S(\mu \mathrm{V} / \mathrm{K})$ and $S^{2} \sigma / \tau\left(\mathrm{W} / \mathrm{mK}^{2} \mathrm{~s}\right)$ are the Seebeck coefficient and the thermoelectric power factor, respectively. A doping level of 0.01 per formula unit corresponds to $\sim 1.552 \times 10^{20} \mathrm{~cm}^{-3}$ for $\mathrm{Mg}_{2} \mathrm{Si}$.

the whole range shown and $T$ in the range 300-750 $\mathrm{K}$ are very similar. For $\mathrm{Mg}_{2} \mathrm{Sn}$ the behavior is also rather symmetric, although less so than $\mathrm{Mg}_{2} \mathrm{Si}$, and in particular while both the $p$-type and $n$-type thermopowers are high at high carrier concentrations $\sim 1-2 \times 10^{20} \mathrm{~cm}^{-3}$ in accord with experiment, the $p$-type thermopowers at given carrier concentration are higher. Thus, from the point of view of the thermopower, $p$-type $\mathrm{Mg}_{2}(\mathrm{Si}, \mathrm{Sn})$ is either similar to or somewhat inferior to $n$ type depending on the composition. By this we mean that the $p$-type $S(T)$ at a given carrier concentration in the range of relevance for thermoelectrics is either similar to or higher than $n$ type at the same carrier concentration.

In order to relate the transport coefficients more directly to the thermoelectric behavior, it is useful to rewrite the figure of merit $Z T=\sigma S^{2} T /\left(\kappa_{l}+\kappa_{e}\right)=r S^{2} / L$, where $r=$ $\kappa_{e} /\left(\kappa_{l}+\kappa_{e}\right)$ and $L=\kappa_{e} /(\sigma T)$, i.e., the Lorenz number in the Wiedemann-Franz relation. The fact that one must have $r \leqslant 1$ implies that the value of the thermopower sets an upper bound on $Z T$. It also shows the doubly detrimental effect of bipolar conduction, which first of all reduces $S$ and secondly increases $L$. Finally, this formula shows the importance of the lattice thermal conductivity in setting the scale for the achievable $Z T$,
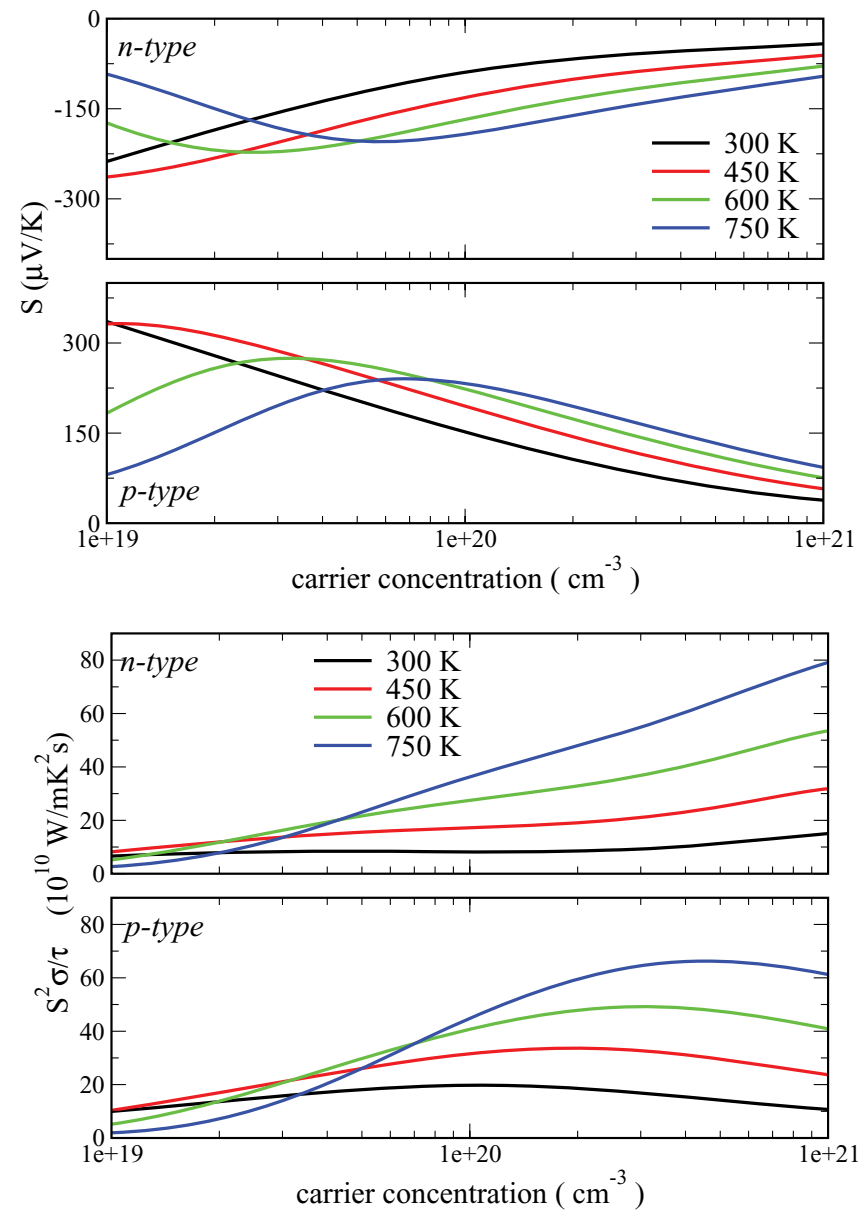

FIG. 7. (Color online) Calculated thermoelectric properties of $\mathrm{Mg}_{2} \mathrm{Sn}$ for both $p$-type and $n$-type charge carriers. The parameters, their units, and the electron and hole carrier concentrations are similar to those defined in Fig. 6. A doping level of 0.01 per formula unit corresponds to $\sim 1.269 \times 10^{20} \mathrm{~cm}^{-3}$ for $\mathrm{Mg}_{2} \mathrm{Sn}$.

since it sets the degree to which the carrier concentration can be lowered to increase $S$ before the lattice thermal conductivity dominates and $r$ becomes small.

In any case, the magnitude of the thermopower in a semiconductor can generally be increased by lowering the carrier concentration until the point where bipolar conduction sets in. However, the optimum thermoelectric performance is generally at higher carrier concentration because of the factor, $r$. In typical high performance thermoelectric materials the highest values of $Z T$ are usually for values of the magnitude of the thermopower in the range 200-300 $\mu \mathrm{V} / \mathrm{K}$ and $r$ of roughly 0.5 . With the standard value of $L=L_{0}, r=0.5$, and $S=250 \mu \mathrm{V} / \mathrm{K}$, this formula gives $Z T \sim 1.3$. Thus the optimum performance is typically at carrier concentrations well above those where the maximum $S$ occurs.

Of the transport coefficients entering $Z T$, the lattice thermal conductivity $\kappa_{l}$ is generally the least dependent on doping. For thermoelectric materials, it typically goes as $1 / T$ until high $T$, where it can saturate near the so-called minimum thermal conductivity, ${ }^{60}$ which is the phonon version of the Ioffe-Regel limit. The electronic component $\kappa_{e}$ is generally proportional to $\sigma$ and $T$ through the Wiedemann-Franz relation. In a degenerate system, where the carrier concentration does not 

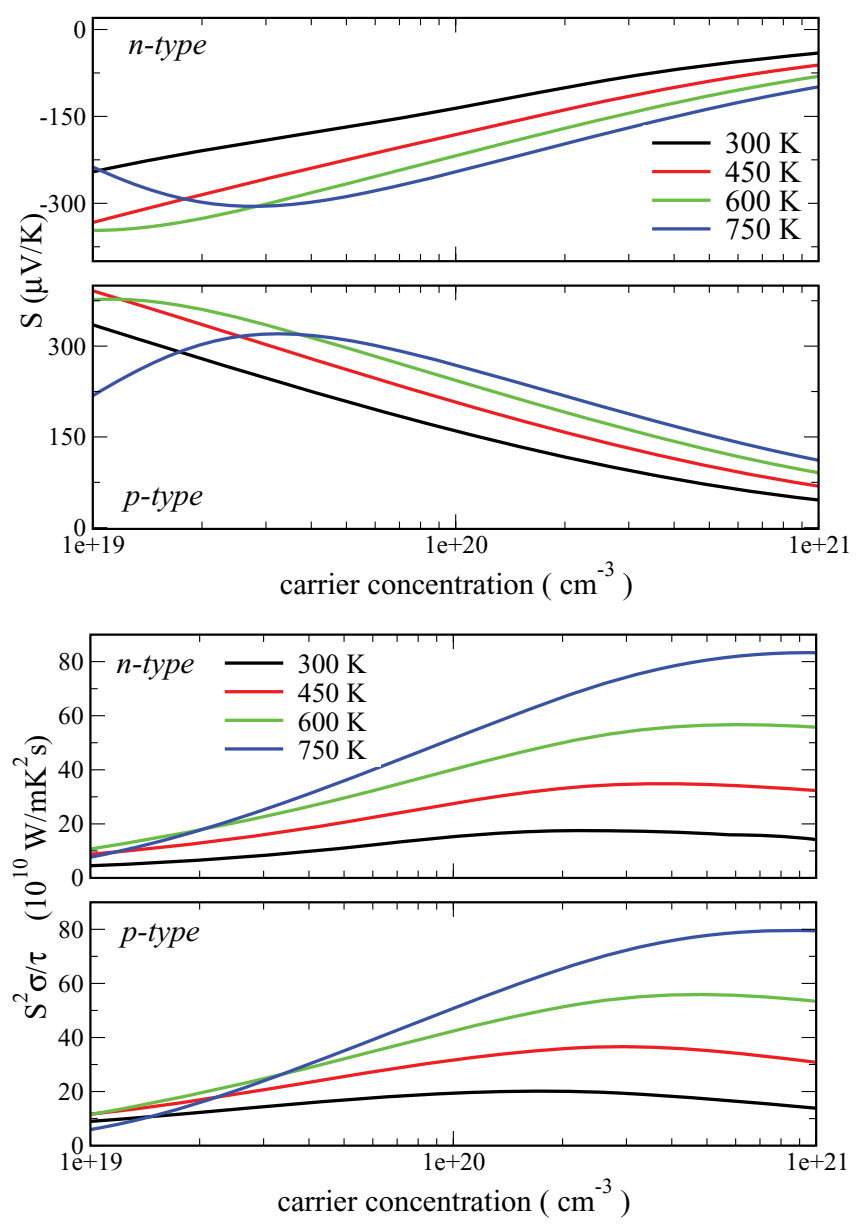

FIG. 8. (Color online) Calculated thermoelectric properties of $\mathrm{Mg}_{2}\left(\mathrm{Si}_{0.5} \mathrm{Sn}_{0.5}\right)$ for both $p$-type and $n$-type charge carriers, as in Fig. 6. A doping level of 0.01 per $\mathrm{Mg}_{2} \mathrm{Si}_{0.5} \mathrm{Sn}_{0.5}$ formula unit corresponds to $\sim 1.395 \times 10^{20} \mathrm{~cm}^{-3}$.

depend on $T$ and where electron phonon scattering dominates the resistivity, $\sigma$ decreases with temperature, usually as $1 / T$ at least for heavy doping levels. In this case, $\kappa_{e}$ is weakly $T$ dependent, $r$ increases with $T$, and $\kappa$ decreases with $T$ but more slowly than the pure lattice $1 / T$ behavior. Reported experimental data for thermoelectric samples of $\mathrm{Mg}_{2}(\mathrm{Si}, \mathrm{Sn})$ with high $Z T$ follow these expectations (note undoped and lightly doped samples show different behavior characteristic of a nondegenerate system with bipolar conduction). ${ }^{3,8-10}$ Therefore we discuss the transport for thermoelectric samples in terms of the above.

The conductivity is given as $\sigma=(\sigma / \tau) \tau$, within the CSTA, where $\sigma / \tau$ is a term that can be calculated directly from the first-principles electronic structure as a function of $T$ and doping level, and $\tau$ is the inverse scattering rate. Calculation of the conductivity requires knowledge of this scattering rate, $\tau^{-1}$, which in general is a function of doping and $T$ and depends on the scattering mechanism. In the electron-phonon regime discussed above, $\tau \propto 1 / T$ (this is the linear in $T$ resistivity of most metals) and also $\tau$ decreases with carrier concentration. In the limit where the lattice thermal conductivity dominates, the function $S^{2} \sigma / \tau$ is an indicator of the behavior of $Z T$, since the $T$ factor in $Z T$ can approximately cancel the $1 / T$ behavior of $1 / \tau$. On the other hand, in the case where the lattice thermal conductivity is small ( $r$ is near unity), $S^{2}$ is the better indicator of the behavior of $Z T$. As mentioned, optimized thermoelectrics are generally intermediate between these two limiting cases, with $S^{2}$ indicating the performance in the overdoped case, and $S^{2} \sigma / \tau$ indicating it in the underdoped case.

The lower panels of Figs. 6-8 show the calculated $S^{2} \sigma / \tau$ as functions of doping and $T$ for the end-point compounds and the 50-50 supercell. Outside the low carrier-concentration bipolar regime, the thermopower increases with $T$, while $\sigma / \tau$ is a weak function of $T$. In any case, for both $p$ type and $n$ type, $\mathrm{Mg}_{2} \mathrm{Si}$ is better than $\mathrm{Mg}_{2} \mathrm{Sn}$ in the carrier concentrations in the range where good thermoelectric performance occurs, i.e., $1 \times 10^{20}-2 \times 10^{20} \mathrm{~cm}^{-3}$, and the supercell shows behavior intermediate between the end points, but the values in this range are closer to those of $\mathrm{Mg}_{2} \mathrm{Sn}$ than $\mathrm{Mg}_{2} \mathrm{Si}$. Importantly, neither indicator of thermoelectric performance shows a clearly enhanced behavior at the intermediate composition, and the interpolation between the end points is similar for $p$ type and $n$ type, even though there is a band crossing as a function of composition for $n$ type, but not for $p$ type.

Turning to the magnitudes of the electronic coefficients, we note that they are similar for $p$ type and $n$ type in all cases. Assuming that the scattering strength is similar for the valence and conduction bands, this would imply that the fully optimized $Z T$ for this material should be similar for $p$ type and $n$ type. Considering that $Z T$ values of $\sim 1.3$ have been reported for $n$ type, we suggest that further optimization of the $p$-type material may yield similar values. The results indicate that the optimum $p$-type carrier concentration will be in a similar range to that of the $n$ type, depending on the exact values of the mobilities that can be achieved in this carrier concentration and $T$ range. If the mobility of the $p$ type is found to be lower than that of the $n$ type for these carrier concentrations, the maximum $Z T$ will be lower, and will occur at higher carrier concentration than the $n$ type, and conversely if the mobility is higher. The fact that the best $p$-type material reported so far has both lower carrier concentration and lower $Z T$ than the best $n$ type thus indicates that it is not optimized for carrier concentration. Finding ways to control and optimize the carrier concentration for $p$ type is therefore an important challenge.

As mentioned, for waste heat recovery from engine exhaust gas, it would be desirable to optimize at a lower $T$ than the temperature where the maximum values of $Z T$ have been reported, i.e., 400-600 K, instead of 700-850 K. ${ }^{8-10}$ The key challenge of going to lower $T$ is the lower values of $S$ that result. In general, this can be addressed by lowering the carrier concentration. However, the extent to which this can be done depends on details, particularly the exact dependence of the thermopower on $T$ and carrier concentration. The results for $\mathrm{Mg}_{2} \mathrm{Si}$ show rather conventional Pisarenko behavior with a near linear dependence of $S$ vs $\log$ carrier concentration. However, for $\mathrm{Mg}_{2} \mathrm{Sn}$ and the 50-50 alloy there is a bipolar effect that is already setting in for both $p$ type and $n$ type at the optimum carrier concentration and $T=750 \mathrm{~K}$. This is less pronounced in the curves for $450 \mathrm{~K}$, meaning that there is more to be gained by lowering the carrier concentration if one desires improved-performance lower $T$.

We also note that the reduction of lattice thermal conductivity due to alloy scattering is more important for lower $T$, since 
at sufficiently high $T$ both the pure compound and the alloy will in principle approach the same minimum thermal conductivity. Thus the use of the alloy to lower thermal conductivity will be more important at $450 \mathrm{~K}$ than at $750 \mathrm{~K}$. Based on the calculated curves for the 50-50 alloy, and assuming that the value of $r$ at the optimum is $\sim 0.5$, as is often the case, we estimate that the optimum carrier concentration for $450 \mathrm{~K}$ is roughly $\sim 50 \%$ of that for the best high-temperature $Z T$.

\section{SUMMARY AND CONCLUSIONS}

Boltzmann transport calculations of the thermopower and $S^{2} \sigma / \tau$ based on the first-principles electronic structures of $\mathrm{Mg}_{2} \mathrm{Si}, \mathrm{Mg}_{2} \mathrm{Sn}$, and a $\mathrm{Mg}_{2}(\mathrm{Si}, \mathrm{Sn})$ supercell show that these materials have rather symmetric behavior with respect to carrier type for carrier concentrations in the range where they show high $Z T$. We find that the electronic transport properties of the supercell are intermediate between those of the end-point compounds. This suggests that, as was emphasized previously, the primary benefit of alloying is through reduction of the thermal conductivity. The results suggest that the performance of a $p$-type alloy can be improved by optimization of the carrier concentration, and that optimization for performance in the range near $450 \mathrm{~K}$ will require a reduction of the carrier concentration by roughly $\sim 50 \%$ from the best value for the best high $T$ performance. Two caveats are that an actual estimation of the maximum $Z T$ for this system depends on detailed knowledge of the $T$ dependent lattice thermal conductivity for the alloy and that we do not know the actual achievable mobility at the operating temperature and carrier concentration for $p$ type. In this regard it is worth noting that the valence bands, which are of importance for $p$ type, have more $\mathrm{Si}-\mathrm{Sn} p$ character than the conduction bands, which may make $p$-type conduction more susceptible to alloy scattering. Since alloy scattering can reduce both thermal conductivity, which is beneficial, and also mobility, which is detrimental, and since the balance is different for $p$ type and $n$ type, it may be that the best $\mathrm{Si}-\mathrm{Sn}$ ratio is different in the two cases. It should also be noted that because the band character is different the strength of the electron phonon scattering for $p$ type could be different from that for $n$ type.

The above results suggest further exploration of the $\mathrm{Si}-\mathrm{Sn}$ alloy system with optimization of the carrier concentration for different ratios of Si to Sn. In particular, the results suggest that higher performance is possible for $p$ type with optimization of the carrier concentration, perhaps at a different $\mathrm{Si}-\mathrm{Sn}$ ratio than for $n$ type.

\section{ACKNOWLEDGMENTS}

S.A. thanks Council of Scientific and Industrial Research (CSIR) - National Physical Laboratory for financial support. The authors gratefully acknowledge use of CSIR High Performance Computing Facilities at CSIR - Centre for Mathematical Modelling and Computer Simulation, Bangalore, India for this research. Work at Oak Ridge National Laboratory was supported by the Department of Energy, Vehicle Technologies, Propulsion Materials Program. Work at CSIR-NPL was supported under TAP-SUN Project (NWP-54).

\footnotetext{
${ }^{1}$ A. F. Ioffe, Semiconductor Thermoelements and Thermoelectric Cooling (Inforsearch, London, 1957).

${ }^{2}$ C. Wood, Rep. Prog. Phys. 51, 459 (1988).

${ }^{3}$ G. J. Snyder and E. S. Toberer, Nat. Mater. 7, 105 (2008).

${ }^{4}$ D. Kraemer, B. Poudel, H. P. Feng, J. C. Caylor, B. Yu, X. Yan, Y. Ma, X. Wang, D. Wang, A. Muto, K. McEnaney, M. Chiesa, Z. Ren, and G. Chen, Nat. Mater. 10, 532 (2011).

${ }^{5}$ B. Poudel, Q. Hao, Y. Ma, Y. Lan, A. Minnich, B. Yu, X. Yan, D. Wang, A. Muto, D. Vashaee, X. Chen, J. Liu, M. S. Dresselhaus, G. Chen, and Z. Ren, Science 320, 634 (2008).

${ }^{6}$ X. Shi, J. Yang, J. R. Salvador, M. Chi, J. Y. Cho, H. Wang, S. Bai, J. Yang, W. Zhang, and L. Chen, J. Am. Chem. Soc. 133, 7837 (2011).

${ }^{7}$ Y. Pei, A. LaLonde, S. Iwanaga, and G. Snyder, Energy Environ. Sci. 4, 2085 (2011)

${ }^{8}$ V. K. Zaitsev, M. I. Fedorov, E. A. Gurieva, I. S. Eremin, P. P. Konstantinov, A. Y. Samunin, and M. V. Vedernikov, Phys. Rev. B 74, 045207 (2006).

${ }^{9}$ Q. Zhang, J. He, T. J. Zhu, S. N. Zhang, X. B. Zhao, and T. M. Tritt, Appl. Phys. Lett. 93, 102109 (2008).

${ }^{10}$ W. Liu, X. Tang, and J. Sharp, J. Phys. D: Appl. Phys. 43, 085406 (2010)

${ }^{11}$ T. Sakamoto, T. Iida, A. Matsumoto, Y. Honda, T. Nemoto, J. Sato, T. Nakajima, H. Taguchi, and Y. Takanashi, J. Electron. Mater. 39, 1708 (2010).

${ }^{12}$ W. Liu, X. Tan, K. Yin, H. Liu, X. Tang, J. Shi, Q. Zhang, and C. Uher, Phys. Rev. Lett. 108, 166601 (2012).

${ }^{13}$ W. J. Luo, M. J. Yang, Q. Shen, H. Y. Jiang, and L. Zhang, Adv. Mater. Res. 66, 33 (2009).

${ }^{14}$ M. Yang, W. Luo, Q. Shen, H. Jiang, and L. Zhang, Adv. Mater. Res. 66, 17 (2009).

${ }^{15}$ H. Gao, T. Zhu, X. Liu, L. Chen, and X. Zhao, J. Mater. Chem. 21, 5933 (2011).

${ }^{16}$ X. Zhang, Q. M. Lu, L. Wang, and F. P. Zhang, J. Electron. Mater. 39, 1413 (2010).

${ }^{17}$ P. Zwolenski, J. Tobola, and S. Kaprzyk, J. Electron. Mater. 40, 889 (2011).

${ }^{18}$ W. H. Fan, R. X. Chen, L. Q. Wang, P. D. Han, and Q. S. Meng, J. Electron. Mater. 40, 1209 (2011).

${ }^{19}$ W. Liu, Q. Zhang, X. F. Tang, H. Li, and J. Sharp, J. Electron. Mater. 40, 1062 (2011).

${ }^{20}$ J. I. Tani and H. Kido, Intermetallics 15, 1202 (2007).

${ }^{21}$ J. I. Tani and H. Kido, Jpn. J. Appl. Phys. 46, 3309 (2007).

${ }^{22}$ G. S. Nolas, D. Wang, and M. Beekman, Phys. Rev. B 76, 235204 (2007).

${ }^{23}$ H. Ihou-Mouko, C. Mercier, J. Tobola, G. Pont, and H. Scherrer, J. Alloys Compd. 509, 6503 (2011).

${ }^{24}$ H. Y. Chen and N. Savvides, J. Electron. Mater. 38, 1056 (2009).

${ }^{25}$ N. Espinosa, M. Lazard, L. Aixala, and H. Scherrer, J. Electron. Mater. 39, 1446 (2010).

${ }^{26}$ D. Parker and D. J. Singh, Phys. Rev. B 82, 035204 (2010).
} 
${ }^{27}$ R. G. Morris, R. D. Redin, and G. C. Danielson, Phys. Rev. 109, 1909 (1958).

${ }^{28}$ M. W. Heller and G. C. Danielson, J. Phys. Chem. Solids 23, 601 (1962).

${ }^{29}$ Q. S. Meng, W. H. Fan, R. X. Chen, and Z. A. Munir, J. Alloys Compd. 509, 7922 (2011).

${ }^{30}$ A. D. Becke and E. R. Johnson, J. Chem. Phys. 124, 221101 (2006).

${ }^{31}$ F. Tran and P. Blaha, Phys. Rev. Lett. 102, 226401 (2009).

${ }^{32}$ D. J. Singh, Phys. Rev. B 82, 205102 (2010).

${ }^{33}$ D. Koller, F. Tran, and P. Blaha, Phys. Rev. B 83, 195134 (2011).

${ }^{34}$ K. P. Ong, D. J. Singh, and P. Wu, Phys. Rev. B 83, 115110 (2011).

${ }^{35}$ D. J. Singh, Phys. Rev. B 82, 205102 (2010).

${ }^{36}$ A. Ghafari, A. Boochani, C. Janowitz, and R. Manzke, Phys. Rev. B 84, 125205 (2011).

${ }^{37}$ W. Feng, D. Xiao, Y. Zhang, and Y. Yao, Phys. Rev. B 82, 235121 (2010).

${ }^{38}$ Y. S. Kim, M. Marsman, G. Kresse, F. Tran, and P. Blaha, Phys. Rev. B 82, 205212 (2010).

${ }^{39}$ D. J. Singh and L. Nordstrom, Planewaves, Pseudopotentials and the LAPW Method, 2nd ed. (Springer, Berlin, 2006).

${ }^{40}$ P. Blaha, K. Schwarz, G. Madsen, D. Kvasnicka, and J. Luitz, WIEN2K, An Augmented Plane Wave + Local Orbitals Program for Calculating Crystal Properties (K. Schwarz, Tech. Univ. Wien, Austria, 2001).

${ }^{41}$ J. P. Perdew, K. Burke, and M. Ernzerhof, Phys. Rev. Lett. 77, 3865 (1996).
${ }^{42}$ G. K. H. Madsen and D. J. Singh, Comput. Phys. Commun. 175, 67 (2006).

${ }^{43}$ D. J. Singh, Sci. Adv. Mater. 3, 561 (2011).

${ }^{44}$ O. Madelung, Numerical Data and Functional Relationships in Science and Technology, Landolt-Börnstein, New Series, Group III Vol. 17e (Springer, Berlin, 1983), pp. 163, 432.

${ }^{45}$ P. Boulet and M. C. Record, J. Chem. Phys. 135, 234702 (2011).

${ }^{46}$ A. Stella, A. D. Brothers, R. H. Hopkins, and D. W. Lynch, Phys. Status Solidi B 23, 697 (1967).

${ }^{47}$ G. Busch and U. Winkler, Physica 20, 1067 (1955).

${ }^{48}$ U. Winkler, Helv. Phys. Acta 28, 633 (1955).

${ }^{49}$ A. Lott and D. W. Lynch, Phys. Rev. 141, 681 (1966).

${ }^{50}$ H. G. Lipson and A. Kahan, Phys. Rev. 133, A800 (1964).

${ }^{51}$ O. Benhelal, A. Chahed, S. Laksari, B. Abbar, B. Bouhafs, and H. Aourag, Phys. Status Solidi B 242, 2022 (2005).

${ }^{52}$ M. Y. Au-Yang and M. L. Cohen, Phys. Rev. 178, 1358 (1969).

${ }^{53}$ B. Arnaud and M. Alouani, Phys. Rev. B 64, 033202 (2001).

${ }^{54}$ D. M. Wood and A. Zunger, Phys. Rev. B 34, 4105 (1986).

${ }^{55}$ A. G. Froseth, R. Hoier, P. M. Derlet, S. J. Andersen, and C. D. Marioara, Phys. Rev. B 67, 224106 (2003).

${ }^{56}$ F. Vazquez, R. A. Forman, and M. Cardona, Phys. Rev. 176, 905 (1968).

${ }^{57}$ H. Takenaka and D. J. Singh, Phys. Rev. B 75, 241102 (2007).

${ }^{58}$ A. Subedi and D. J. Singh, Phys. Rev. B 78, 045106 (2008).

${ }^{59}$ D. J. Singh and I. I. Mazin, Phys. Rev. B 56, R1650 (1997).

${ }^{60}$ D. G. Cahill, S. K. Watson, and R. O. Pohl, Phys. Rev. B 46, 6131 (1992). 